J. Nonlinear Var. Anal. 2 (2018), No. 3, pp. 379-389

Available online at http://jnva.biemdas.com

https://doi.org/10.23952/jnva.2.2018.3.10

\title{
OPTIMALITY CONDITIONS FOR WEAKLY EFFICIENT SOLUTIONS OF VECTOR VARIATIONAL INEQUALITIES VIA CONVEXIFICATORS
}

\author{
TRAN THI MAI ${ }^{1}$, DO VAN LUU ${ }^{2,3, *}$ \\ ${ }^{1}$ Thai Nguyen University of Economics and Business Administration, Vietnam \\ ${ }^{2}$ TIMAS, Thang Long University, Hanoi, Vietnam \\ ${ }^{3}$ Institute of Mathematics, Vietnam Academy of Science and Technology, Hanoi, Vietnam
}

\begin{abstract}
Fritz John necessary conditions for weakly efficient solutions of nonsmooth vector variational inequality problems with constraints in terms of convexificators are derived. Karush-Kuhn-Tucker necessary efficiency conditions are established under a Mangasarian-Fromovitz type constraint qualification. Finally, sufficient conditions are given under suitable assumptions on the generalized convexity.

Keywords. Vector variational inequality; Weakly efficient solution; Fritz John and Karush-Kuhn-Tucker efficiency conditions; Convexificators; Locally Lipschitz functions.
\end{abstract}

2010 Mathematics Subject Classification. 90C46, 91B50, 49J52.

\section{INTRODUCTION}

In recent years, vector variational inequalities have extensively and broadly studied because of their fields of applications. Optimality conditions for vector variational inequality problems have been studied by many authors; see, e.g., [1, 2, 5, 6, 10, 11, 14, 18, 19] and references therein. Giannessi, Mastroeni and Pellegrini [5] derived sufficient optimality conditions for efficiency of vector variational inequalities in finite dimension. Morgan and Romaniello [14] gave the Kuhn-Tucker conditions for weak vector generalized quasivariational inequalities with inequality constraints. Optimality conditions are obtained in $[18,19]$ by establishing the equivalence between a vector variational inequality and a vector optimization problem. Luu and Hang [10] derived optimality conditions for weak efficient, global efficient, and efficient solutions of constraints vector variational inequalities via the Clarke and Michel-Penot subdifferential. The notion of convexificator provides good calculus rules for establishing optimality conditions in nonsmooth optimization. The notion of convex and compact convexificator was first introduced by Demyanov [4]. Jeyakumar and Luc [9] introduced the notion of closed and nonconvex convexificators for extended-real-valued functions; see [9] and the references therein. The notion of convexificator is a generalization of some notions of known subdifferentials such as the subdifferentials of Clarke [3], Michel-Penot [15], Mordukhovich [16]. Optimality conditions for efficiency via convexificators have been developed by many authors. Recently, by using this notion, Luu [11, 12, 13] derived Lagrange multiplier rules for efficiency, and Golestani and Nobakhtian [7] established strong Karush-Kuhn-Tucker

${ }^{*}$ Corresponding author.

E-mail addresses: tranthimai879@gmail.com (T.T. Mai), dvluu@math.ac.vn (D.V. Luu).

Received July 24, 2018; Accepted September 3, 2018.

(C) 2018 Journal of Nonlinear and Variational Analysis 
conditions. Luu [11] established Fritz John and Karush-Kuhn-Tucker necessary conditions for local efficient solutions of constrained vector equilibrium problems in Banach spaces via convexificators.

The paper is organized as follows. In Section 2, necessary preliminaries are provided. Section 3 deals with Fritz John necessary conditions for weakly efficient solutions of nonsmooth vector variational inequality problems with constraints in terms of convexificators. Note that the problem studied here involves a cone-constraint with respect to a polyhedral convex cone, and weakly efficient solutions are considered with respect to pointed, closed and convex cones. Section 4 will be devoted to developing Karush-Kuhn-Tucker necessary conditions for weakly efficient solutions of constrained vector variational inequalities under a Mangasarian-Fromovitz type constraint qualification. In Section 5, sufficient conditions are given under suitable assumptions on generalized convexity.

\section{PRELIMINARIES}

Let $X$ be a real Banach space, and let $X^{*}$ be the topological dual of $X$. Let $C$ be a closed subset of $X$. Let $g$ and $h$ be maps from $X$ into $\mathbb{R}^{m}$ and $\mathbb{R}^{l}$, respectively. $g=\left(g_{1}, \ldots, g_{m}\right), h=\left(h_{1}, \ldots, h_{l}\right)$ with $g_{i}, h_{j}(i=1, \ldots, m ; j=1, \ldots, l)$ are extended-real-valued functions defined on $X$. Assume that $g$ is locally Lipschitz at $\bar{x} \in C$, and $S$ is a polyhedral convex cone in $\mathbb{R}^{m}$. We set

$$
M:=\{x \in C: g(x) \in S, h(x)=0\} .
$$

Sine $S$ is a polyhedral convex cone in $\mathbb{R}^{m}$, it follows that $S$ is of the form

$$
S=\left\{y \in \mathbb{R}^{m}:\left\langle a_{i}, y\right\rangle \geqslant 0, i=1, \ldots, r\right\}\left(a_{i} \in \mathbb{R}^{m}, i=1, \ldots, r\right) .
$$

Putting

$$
\widetilde{g}_{i}(x)=-\left\langle a_{i}, g(x)\right\rangle(i=1, \ldots, r),
$$

it follows from (1) that

$$
g(x) \in S \Longleftrightarrow \widetilde{g}_{i}(x) \leqslant 0(i=1, \ldots, r) .
$$

So, the set $M$ has the following form:

$$
M=\left\{x \in C: \widetilde{g}_{i}(x) \leqslant 0(i=1, \ldots, r), h_{j}(x)=0(j \in L:=\{1, \ldots, l\})\right\} .
$$

For $\bar{x} \in M$, we set

$$
I(\bar{x})=\left\{i \in\{1, \ldots, r\}: \widetilde{g}_{i}(x)=0\right\} .
$$

Recall that a point $\bar{x}$ is said to be a regular point in the sense of Ioffe for $h$ relative to $C$ [8] if there exist numbers $K>0$ and $\delta>0$ such that, for all $x \in C \cap B(\bar{x} ; \delta)$,

$$
d_{H}(x) \leqslant K\|h(x)-h(\bar{x})\|,
$$

where $H:=\{x \in C: h(x)=h(\bar{x})\}, d_{H}(x)$ denotes the distance from $x$ to $H, B(\bar{x} ; \delta)$ stands for the open ball of radius $\delta$ around $\bar{x}$.

The lower and upper Dini derivatives of $f: X \longrightarrow \overline{\mathbb{R}}=\mathbb{R} \cup\{ \pm \infty\}$ at $\bar{x} \in X$ in the direction $v$ are defined respectively by

$$
f_{d}^{-}(\bar{x} ; v)=\liminf _{t \downarrow 0} \frac{f(\bar{x}+t v)-f(\bar{x})}{t}
$$

and

$$
f_{d}^{+}(\bar{x} ; v)=\limsup _{t \downarrow 0} \frac{f(\bar{x}+t v)-f(\bar{x})}{t} .
$$


Following [9], the function $f: X \rightarrow \overline{\mathbb{R}}$ is said to have an upper (lower) convexificator $\partial^{*} f(x)$ (resp. $\left.\partial_{*} f(x)\right)$ at $\bar{x}$ if $\partial^{*} f(\bar{x}) \subset X^{*}$ (resp. $\partial_{*} f(\bar{x}) \subset X^{*}$ ) is weakly* closed and, for all $v \in X$,

$$
\begin{gathered}
f_{d}^{-}(\bar{x}, v) \leq \sup _{x^{*} \in \partial^{*} f(x)}\left\langle x^{*}, v\right\rangle \\
\text { (resp. } \left.f_{d}^{+}(\bar{x}, v) \geq \inf _{x^{*} \in \partial_{*} f(x)}\left\langle x^{*}, v\right\rangle\right) .
\end{gathered}
$$

The function $f: X \rightarrow \overline{\mathbb{R}}$ is said to have a convexificator $\partial^{*} f(x)$ at $\bar{x}$ if it is both upper and lower convexificator of $f$ at $\bar{x}$. This means that, for each $v \in X$,

$$
\begin{aligned}
& f_{d}^{-}(x, v) \leq \sup _{x^{*} \in \partial^{*} f(x)}\left\langle x^{*}, v\right\rangle, \\
& f_{d}^{+}(x, v) \geq \inf _{x^{*} \in \partial_{*} f(x)}\left\langle x^{*}, v\right\rangle .
\end{aligned}
$$

The function $f$ is said to have an upper (lower) semi-regular convexificator $\partial^{*} f(x)$ (resp. $\partial_{*} f(x)$ ) at $\bar{x}$ if $\partial^{*} f(\bar{x})$ (resp. $\partial_{*} f(\bar{x})$ ) is weakly* closed and for all $v \in X$,

$$
\begin{gathered}
f_{d}^{+}(\bar{x}, v) \leq \sup _{x^{*} \in \partial^{*} f(x)}\left\langle x^{*}, v\right\rangle \\
\text { (resp. } \left.f_{d}^{-}(\bar{x}, v) \geq \inf _{x^{*} \in \partial^{*} f(x)}\left\langle x^{*}, v\right\rangle\right) .
\end{gathered}
$$

If equality holds in (2.2) (resp. (2.3)), then $\partial^{*} f(\bar{x})$ (resp. $\partial_{*} f(\bar{x})$ ) is called an upper (resp. lower) regular convexificator of $f$ at $\bar{x}$.

Let $f$ be a real-valued function defined on $X$. Following [3], the Clarke directional derivative of $f$ at $\bar{x}$ with respect to the direction $v$ is defined as

$$
f^{0}(\bar{x}, v)=\limsup _{x \rightarrow \bar{x}, t \downarrow 0} \frac{f(x+t v)-f(x)}{t},
$$

The Clarke subdifferential of $f$ at $\bar{x}$ is

$$
\partial f(\bar{x})=\left\{x^{*} \in X^{*}:\left\langle x^{*}, v\right\rangle \leq f^{\circ}(\bar{x}, v), \forall v \in X\right\} .
$$

where $\langle.,$.$\rangle denotes the coupling between X^{*}$ and $X$. If $f$ is locally Lipschitz, then the Clarke subdifferential is a convexificator of $f$ at $\bar{x}$; see [9] and the references therein.

Recall that a locally Lipschitz function $f$ is called regular in the sense of Clarke at $\bar{x}[3]$ if, for every $v \in$ $X$, there exist $f^{\prime}(\bar{x} ; v)$ and $f^{\prime}(\bar{x} ; v)=f^{0}(\bar{x} ; v)$. For such function $f$, the Clarke subdifferential $\partial f(\bar{x})$ is an upper regular convexificator and the convexificator map $\partial f$ is locally bounded at $\bar{x}$ (see [[9], Corollary 5.2]). Moreover, in case $\operatorname{dim} X<\infty$, the map $\partial f$ is upper semicontinuous at $\bar{x}$ (see [3]).

If $f$ is a convex function on $X$, then the subdifferential of $f$ at $\bar{x}$ is defined as

$$
\partial_{C} f(\bar{x}):=\left\{\xi \in X^{*}:\langle\xi, x-\bar{x}\rangle \leqslant f(x)-f(\bar{x}), \forall x \in X\right\} .
$$

Proposition 7.3.9 [17] pointed that if $f$ is convex on $X$ and locally Lipschitz at $\bar{x} \in X$, then

$$
\partial_{C} f(\bar{x})=\partial f(\bar{x})
$$

The Clarke tangent cone to a set $C$ at $\bar{x} \in C$ is defined as

$$
\begin{gathered}
T_{C}(\bar{x}):=\left\{v \in X: \forall x_{n} \in C, x_{n} \rightarrow \bar{x}, \forall t_{n} \downarrow 0, \exists v_{n} \rightarrow v\right. \\
\text { such as } \left.x_{n}+t_{n} v_{n} \in C, \forall n\right\} .
\end{gathered}
$$


The Clarke normal cone $C$ at $\bar{x}$ is

$$
N_{C}(\bar{x}):=\left\{\xi \in X^{*}:\langle\xi, v\rangle \leqslant 0, \forall v \in T_{C}(\bar{x})\right\}
$$

Proposition 2.1. [9] Suppose that the function $f: X \rightarrow \mathbb{R}$ admits an upper convexificator $\partial^{*} f(\bar{x})$ at $\bar{x} \in X$. If $f$ attains its minimum at $\bar{x}$, then

$$
0 \in \operatorname{clconv} \partial^{*} f(\bar{x})
$$

where cl and conv indicate the weak* closure and the convex hull, respectively.

Proposition 2.2. [9] Let $f=\left(f_{1}, \ldots f_{n}\right)$ be a continuous functions from $X$ to $\mathbb{R}^{n}$, and let $g: \mathbb{R}^{n} \rightarrow \mathbb{R}$ be a continuous function. Suppose that, for each $i=1, \ldots n, f_{i}$ admits a bounded convexificator $\partial^{*} f_{i}(\bar{x})$ at $\bar{x}$ and $g$ admits a bounded convexificator $\partial^{*} g(f(\bar{x}))$ at $f(\bar{x})$. For each $i=1, \ldots n$, if $\partial^{*} f_{i}$ is upper semicontinuous at $\bar{x}$ and $\partial^{*} g$ is upper semicontinuous at $f(\bar{x})$, then the set

$$
\partial^{*}(g \circ f)(\bar{x})=\partial^{*} g(f(\bar{x}))\left(\partial^{*} f_{1}(\bar{x}), \ldots, \partial^{*} f_{n}(\bar{x})\right)
$$

is a convexificator of $g \circ f$ at $\bar{x}$.

\section{Fritz John Necessary CONDitions fOR WEAKly EFFicient SOlutions}

Let $L\left(X, \mathbb{R}^{p}\right)$ be the space of all continuous linear mappings from $X$ to $\mathbb{R}^{p}$, and let $T$ be a mapping from $X$ to $L\left(X, \mathbb{R}^{p}\right)$. Let $Q$ be a pointed, closed and convex cone in $\mathbb{R}^{p}$ with nonempty interior. Let us consider the following vector variational inequality (VVI): Find a point $\bar{x} \in M$ such as

$$
T(\bar{x})(y-\bar{x}) \notin-\operatorname{int} Q, \quad \forall y \in M .
$$

A vector $\bar{x}$ is called a weakly efficient solution of (VVI) if (3.1) holds. Note that in case int $Q=\mathbb{R}_{++}^{p}$, the definition of weakly efficient solution is of the form: There is no $y \in M$ such that

$$
T(\bar{x})_{k}(y-\bar{x})<0, \quad \forall k \in J:=\{1, \ldots, p\},
$$

where $T(\bar{x})=\left(T(\bar{x})_{1}, \ldots, T(\bar{x})_{p}\right), T(\bar{x})_{k}: X \rightarrow \mathbb{R}(\forall k \in J), \mathbb{R}_{++}^{p}=\operatorname{int} \mathbb{R}_{+}^{p}$.

To derive Fritz John necessary conditions for a weak efficient solution $\bar{x}$ of Problem (VVI), we introduce the following assumptions.

Algorithm 3.1. The functions $h_{1}, \ldots, h_{l}$ are locally Lipschitz at $\bar{x}$; for each $j \in L$, the function $\left|h_{j}\right|$ is regular in the sense of Clarke at $\bar{x}$, and the convexificator map $\partial^{*} h_{j}$ is upper semicontinuous at $\bar{x} ; g_{i}(i \in$ $I(\bar{x}))$ are continuous, and $\widetilde{g}_{i}(i \in I(\bar{x}))$ admit upper convexificators $\partial^{*} \widetilde{g}_{i}(\bar{x})$ at $\bar{x} ; C$ is convex.

We shall begin with establishing a Fritz John necessary condition for weak efficient solutions of problem (VVI).

Theorem 3.1. Let $\bar{x}$ be a weakly efficient solution of (VVI). Assume that $\bar{x}$ is a regular point in the sense of Ioffe for $h$ relative to $C$, and Assumption 3.1 is fulfilled. Then, there exist $\bar{\theta} \geqslant 0, \bar{\chi}:=\left(\bar{\chi}_{1}, \ldots, \bar{\chi}_{p}\right) \in$ $Q^{*} \backslash\{0\}, \bar{\mu}_{i} \geqslant 0(\forall i \in I(\bar{x})), \bar{\gamma}_{j} \in \mathbb{R}(j=1, \ldots, l)$ such that $\bar{\theta}+\sum_{i \in I(\bar{x})} \bar{\mu}_{i}=1$, and

$$
0 \in \operatorname{cl}\left(\sum_{k \in J} \bar{\theta} \bar{\chi}_{k} T(\bar{x})_{k}+\sum_{i \in I(\bar{x})} \bar{\mu}_{i} \operatorname{conv} \partial^{*} \widetilde{g}_{i}(\bar{x})+\sum_{j \in L} \bar{\gamma}_{j} \operatorname{conv} \partial^{*} h_{j}(\bar{x})+N_{C}(\bar{x})\right) .
$$


Proof. Putting $F(\bar{x}, y):=T(\bar{x})(y-\bar{x})$, we have $F(\bar{x},$.$) is an affine map,$

$$
F:=\left(F_{1}, \ldots, F_{p}\right): X \rightarrow \mathbb{R}^{p}
$$

and $F(\bar{x}, \bar{x})=0$. We set $F_{\bar{x}}(y):=F(\bar{x}, y)$, and

$$
F_{k, \bar{x}}(y):=F_{k}(\bar{x}, y)=T(\bar{x})_{k}(y-\bar{x})(k \in J) .
$$

Since $T(\bar{x})($.$) is continuous linear, it is strictly differentiable and locally Lipschitz. Hence, the function$ $F_{k, \bar{x}}().(\forall k \in J)$ admit an upper convexificator at $\bar{x}$ as $\left\{T(\bar{x})_{k}\right\}(\forall k \in J)$.

Hence, $\bar{x}$ is a weakly efficient solution of the multiobjective optimization problem (MP):

$$
\min F_{\bar{x}}(y) \text { s.t. } y \in M:=\left\{y \in C: \widetilde{g}_{i}(y) \leqslant 0(i=1, \ldots, r), h_{j}(y)=0(j=1, \ldots, l)\right\} .
$$

Consequently, $\bar{x}$ is a weakly efficient solution of the vector equilibrium problem (VEP):

$$
F(\bar{x}, y) \notin-\text { int } Q \text { s.t. } y \in M=\left\{y \in C: \widetilde{g}_{i}(y) \leqslant 0(i=1, \ldots, r), h_{j}(y)=0(j=1, \ldots, l)\right\} .
$$

Applying the scalarization theorem by Gong ([6], Theorem 3.1) to Problem (VEP) yields the existence of a continuous positively homogeneous subadditive function $P$ on $\mathbb{R}^{p}$ satisfying the property

$$
y_{2}-y_{1} \in \operatorname{int} Q \Longrightarrow P\left(y_{1}\right)<P\left(y_{2}\right),
$$

and

$$
\left(P \circ F_{\bar{x}}\right)(y) \geqslant 0(\forall y \in M) .
$$

Hence, $\bar{x}$ is a solution of Problem (SP):

$$
\min \left(P \circ F_{\bar{x}}\right)(x) \text { s.t. } x \in M=\left\{x \in C: \widetilde{g}_{i}(x) \leqslant 0(i=1, \ldots, r), h_{j}(x)=0(j \in L)\right\} .
$$

We invoke Theorem 3.2 in [11] to Problem (SP) to deduce that there exist $\bar{\theta} \geqslant 0, \bar{\mu}_{i}(\forall i \in I(\bar{x})), \bar{\gamma}_{j} \in$ $\mathbb{R}(j \in J)$ such that $\bar{\theta}+\sum_{i \in I(\bar{x})} \bar{\mu}_{i}=1$, and

$$
\begin{gathered}
0 \in \operatorname{cl}\left(\bar{\theta} \partial^{*}\left(P \circ F_{\bar{x}}\right)(\bar{x})\right)+\sum_{i \in I(\bar{x})} \bar{\mu}_{i} \operatorname{conv} \partial^{*} \widetilde{g}_{i}(\bar{x}) \\
\left.+\sum_{j \in L} \bar{\gamma}_{j} \operatorname{conv}^{*} h_{j}(\bar{x})+N_{C}(\bar{x})\right) .
\end{gathered}
$$

We now check the hypotheses of a chain rule by Jeyakumar-Luc ([9], Proposition 5.1) to the composite function

$$
\left(P \circ F_{\bar{x}}\right)(x)=(P \circ(f-f(\bar{x}))(x)=(P \circ f)(x) .
$$

Since $P$ is continuous convex, we can apply Proposition 2.2.6 [3] to deduce that it is locally Lipschitz. Hence, its subdifferential $\partial_{C} P(f(x))$ is a bounded convexificator of $P$ at $f(\bar{x})=0$. Since $P$ is convex and locally Lipschitz, Proposition 7.3.9 [17] was pointed that

$$
\partial_{C} P(f(\bar{x}))=\partial P(f(\bar{x})) .
$$

Note that

$$
\partial f(\bar{x})=\{T(\bar{x})\}, \quad \forall k \in J
$$

and $f_{k}$ admits an upper convexificator at $\bar{x}$ as $\left\{T(\bar{x})_{k}\right\}(\forall k \in J)$ and $f(\bar{x})=0$. Moreover, the Clarke subdifferential map $\partial P$ is upper semicontinuous at $f(\bar{x})=0$. By virtue of Proposition 5.1 [9], the following set:

$$
\partial_{C} P(0)\left(T(\bar{x})_{1}, \ldots, T(\bar{x})_{p}\right)
$$


is a convexificator of $P \circ f$ at $\bar{x}$. Hence, (3.3) is of the form:

$$
\begin{gathered}
0 \in \operatorname{cl}\left(\bar{\theta} \partial_{C} P(0)\left(T(\bar{x})_{1}, \ldots, T(\bar{x})_{p}\right)+\sum_{i \in I(\bar{x})} \bar{\mu}_{i} \operatorname{conv} \partial^{*} \widetilde{g}_{i}(\bar{x})+\right. \\
\left.+\sum_{j \in L} \bar{\gamma}_{j} \operatorname{conv} \partial^{*} h_{j}(\bar{x})+N_{C}(\bar{x})\right) .
\end{gathered}
$$

It follows from (3.4) that there exist

$$
\begin{gathered}
z_{n} \in \bar{\theta} \partial_{C} P(0)\left(T(\bar{x})_{1}, \ldots, T(\bar{x})_{p}\right)+\sum_{i \in I(\bar{x})} \bar{\mu}_{i} \operatorname{conv} \partial^{*} \widetilde{g}_{i}(\bar{x}) \\
+\sum_{j \in L} \bar{\gamma}_{j} \operatorname{conv} \partial^{*} h_{j}(\bar{x})+N_{C}(\bar{x})
\end{gathered}
$$

such that $\lim _{n \rightarrow \infty} z_{n}=0$. By (3.5), there exist a sequence $\left\{\chi_{n}\right\} \subset \partial_{C} P(0) \subset \mathbb{R}^{m}$, such that

$$
\begin{gathered}
z_{n} \in \bar{\theta} \chi_{n}\left(T(\bar{x})_{1}, \ldots, T(\bar{x})_{p}\right)+\sum_{i \in I(\bar{x})} \bar{\mu}_{i} \operatorname{conv} \partial^{*} \widetilde{g}_{i}(\bar{x}) \\
+\sum_{j \in L} \bar{\gamma}_{j} \operatorname{conv} \partial^{*} h_{j}(\bar{x})+N_{C}(\bar{x}) .
\end{gathered}
$$

Since $\partial_{C} P(0)$ is a compact set in $\mathbb{R}^{p}$, without loss of generality, we can assume that

$$
\chi_{n} \rightarrow \bar{\chi}:=\left(\bar{\chi}_{1}, \ldots, \bar{\chi}_{p}\right) \in \partial_{C} P(0)
$$

Using (3.3) and (3.6), we find that

$$
0 \in \operatorname{cl}\left(\sum_{k \in J} \bar{\theta} \bar{\chi}_{k} T(\bar{x})_{k}+\sum_{i \in I(\bar{x})} \bar{\mu}_{i} \operatorname{conv} \partial^{*} \widetilde{g}_{i}(\bar{x})+\sum_{j \in L} \bar{\gamma}_{j} \operatorname{conv} \partial^{*} h_{j}(\bar{x})+N_{C}(\bar{x})\right) .
$$

Let us see that $\bar{\chi} \in Q^{*} \backslash\{0\}$. In fact, for any $y \in \operatorname{int} Q$, it can be written $0-(-y) \in \operatorname{int} Q$. Since $\bar{\chi} \in$ $\partial P(f(\bar{x}))$ and $f(\bar{x})=0$, we arrive at

$$
\begin{aligned}
\langle\bar{\chi},-y\rangle & =\langle\bar{\chi},(f(\bar{x})-y)-f(\bar{x})\rangle \\
& \leq P(f(\bar{x})-y)-P(f(\bar{x})) \\
& =P(-y) \\
& <P(0)=0 .
\end{aligned}
$$

Consequently, $\bar{\chi} \in Q \backslash\{0\}$. The proof is complete.

\section{Karush-Kuhn-Tucker Necessary Conditions For WeAK EFFicient Solutions}

In this section, the following Mangasarian-Fromovitz type constraints qualification (MFCQ) is introduced in order to derive Karush-Kuhn-Tucker necessary conditions for weak efficient solutions of Problem (VVI): There exist $v_{0} \in T_{C}(\bar{x})$ and numbers $a_{i}>0(i \in I(\bar{x}))$ such that

(1) $\left\langle\xi_{i}, v_{0}\right\rangle \leqslant-a_{i}, \quad \forall \xi_{i} \in \widetilde{\partial}^{*} g_{i}(\bar{x}), \forall i \in I(\bar{x})$;

(2) $\left\langle\eta_{j}, v_{0}\right\rangle=0, \quad \forall \eta_{j} \in \partial^{*} h_{j}(\bar{x}), \forall j \in L$.

A Karush-Kuhn-Tucker necessary condition for weakly efficient solutions of Problem (VVI) can be stated as follows. 
Theorem 4.1. Let $\bar{x}$ be a weakly efficient solution of (VVI). Assume all hypotheses of Theorem 3.1 and the constraint qualification (MFCQ) hold. Then there exist $\bar{\lambda}:=\left(\bar{\lambda}_{1}, \ldots, \bar{\lambda}_{p}\right) \in Q^{*} \backslash\{0\}, \bar{\mu}_{i} \geqslant 0(\forall i \in$ $I(\bar{x})), \bar{\gamma}_{j} \in \mathbb{R}(j=1, \ldots, l)$ such that

$$
0 \in \operatorname{cl}\left(\sum_{k \in J} \bar{\lambda}_{k} T(\bar{x})_{k}+\sum_{i \in I(\bar{x})} \bar{\mu}_{i} \operatorname{conv} \partial^{*} \widetilde{g}_{i}(\bar{x})+\sum_{j \in L} \bar{\gamma}_{j} \operatorname{conv} \partial^{*} h_{j}(\bar{x})+N_{C}(\bar{x})\right) .
$$

Proof. From Theorem 3.1, we find that there exist $\bar{\theta} \geqslant 0, \bar{\chi}:=\left(\bar{\chi}_{1}, \ldots, \bar{\chi}_{p}\right) \in Q^{*} \backslash\{0\}, \bar{\mu}_{i} \geqslant 0(\forall i \in$ $I(\bar{x})), \bar{\gamma}_{j} \in \mathbb{R}(j=1, \ldots, l)$ such that

$$
\bar{\theta}+\sum_{i \in I(\bar{x})} \bar{\mu}_{i}=1
$$

and

$$
0 \in \operatorname{cl}\left(\sum_{k \in J} \bar{\theta} \bar{\chi}_{k} T(\bar{x})_{k}+\sum_{i \in I(\bar{x})} \bar{\mu}_{i} \operatorname{conv} \partial^{*} \widetilde{g}_{i}(\bar{x})+\sum_{j \in L} \bar{\gamma}_{j} \operatorname{conv} \partial^{*} h_{j}(\bar{x})+N_{C}(\bar{x})\right) .
$$

Putting $\bar{\lambda}:=\bar{\theta} \bar{\chi}$, we obtain (4.1) immediately. Next, we prove that $\bar{\lambda} \in Q^{*} \backslash\{0\}$. To do this, we show $\bar{\theta}>0$. Assume the contrary that $\bar{\theta}=0$. Then $\sum_{i \in I(\bar{x})} \bar{\mu}_{i}=1$. There exist

$$
\begin{gathered}
\xi_{i}^{(n)} \in \operatorname{conv}^{*} \widetilde{g}_{i}(\bar{x}), \quad \forall i \in I(\bar{x}), \\
\eta_{j}^{(n)} \in \operatorname{conv} \partial^{*} h_{j}(\bar{x}), \quad \forall j \in L
\end{gathered}
$$

and $\zeta^{(n)} \in N_{C}(\bar{x})$ such that

$$
0=\lim _{n \rightarrow \infty}\left[\sum_{i \in I(\bar{x})} \bar{\mu}_{i} \xi_{i}^{(n)}+\sum_{j \in L} \bar{\gamma}_{j} \eta_{j}^{(n)}+\zeta^{(n)}\right]
$$

For $v_{0} \in T_{C}(\bar{x})$, we find from (4.2) that

$$
0=\lim _{n \rightarrow \infty}\left[\sum_{i \in I(\bar{x})} \bar{\mu}_{i}\left\langle\xi_{i}^{(n)}, v_{0}\right\rangle+\sum_{j \in L} \bar{\gamma}_{j}\left\langle\eta_{j}^{(n)}, v_{0}\right\rangle+\left\langle\zeta^{(n)}, v_{0}\right\rangle\right] .
$$

Note that $\sum_{i \in I(\bar{x})} \bar{\mu}_{i}=1$. Taking account of (MFCQ) and (4.3), we get

$$
\begin{aligned}
0= & \lim _{n \rightarrow \infty}\left[\sum_{i \in I(\bar{x})} \bar{\mu}_{i}\left\langle\xi_{i}^{(n)}, v_{0}\right\rangle+\sum_{j \in L} \bar{\gamma}_{j}\left\langle\eta_{j}^{(n)}, v_{0}\right\rangle+\left\langle\zeta^{(n)}, v_{0}\right\rangle\right] \\
& \leqslant \lim _{n \rightarrow \infty}\left[\sum_{i \in I(\bar{x})} \bar{\mu}_{i}\left\langle\xi_{i}^{(n)}, v_{0}\right\rangle+\sum_{j \in L} \bar{\gamma}_{j}\left\langle\eta_{j}^{(n)}, v_{0}\right\rangle\right] \\
& \leqslant-\sum_{i \in I(\bar{x})} \bar{\mu}_{i} a_{i} \\
& <0
\end{aligned}
$$

which is a contradiction. Hence $\bar{\theta}>0$. The latter along with the fact that $\bar{\chi} \in Q^{*} \backslash\{0\}$ yields that $\bar{\lambda} \in Q^{*} \backslash\{0\}$. This completes the proof.

Next, we illustrate Theorem 4.1 with the following example.

Example 4.1. Let $X=\mathbb{R}^{2}, Y=\mathbb{R}^{2}, C=[0,1] \times[-1,0], Q=\left\{\left(x_{1}, x_{2}\right) \in \mathbb{R}^{2}:\left|x_{2}\right| \leqslant \frac{3}{4} x_{1}\right\}$, and $\bar{x}=(0,0)$. Let $T: \mathbb{R}^{2} \rightarrow L\left(\mathbb{R}^{2}, \mathbb{R}^{2}\right)$ be defined as

$$
T(x)=\left(\begin{array}{ll}
a(x) & b(x) \\
c(x) & d(x)
\end{array}\right),
$$


where

$$
\begin{aligned}
& a(x)=\frac{1}{2}\left|x_{1}-1\right|+\left|x_{2}\right|, b(x)=e^{x_{2}}+x_{1}^{2}, \\
& c(x)=\sin x_{1}+3 \cos x_{2}, d(x)=\left(x_{1}-1\right)\left(x_{2}+2\right), \\
& \left(\left(x_{1}, x_{2}\right) \in \mathbb{R}^{2}\right) .
\end{aligned}
$$

Then $a(\bar{x})=1 / 2, b(\bar{x})=1, c(\bar{x})=3$, and $d(\bar{x})=-2$. Thus, $T(\bar{x})=\left(\begin{array}{cc}\frac{1}{2} & 1 \\ 3 & -2\end{array}\right)$ and, for $x=\left(x_{1}, x_{2}\right) \in \mathbb{R}^{2}$,

$$
\begin{aligned}
& T(\bar{x})_{1}(x)=\frac{1}{2} x_{1}+x_{2}, \\
& T(\bar{x})_{2}(x)=3 x_{1}-2 x_{2} .
\end{aligned}
$$

Let $g, h$ be defined as $g:=\left(g_{1}, g_{2}\right)$, where

$$
g_{1}(x):= \begin{cases}x_{1}^{2}\left(1-\sin \frac{1}{x_{1}}\right)+2 x_{1}, & \text { if } \quad x_{1} \neq 0 \\ 0, & \text { if } \quad x_{1}=0\end{cases}
$$

$g_{2}(x):=x_{2}^{2}-\left|x_{2}\right|+\frac{3}{2} x_{2}$, and $h(x):=\frac{1}{3} x_{1}+\frac{1}{2} x_{2}$. From the notations in Section 2, we have

$$
S=\left\{\left(x_{1}, x_{2}\right) \in \mathbb{R}^{2}: x_{1} \geq 0, x_{2} \leq 0\right\},
$$

$a_{1}=(1,0), a_{2}=(0,-1)$, and

$$
\begin{aligned}
& \widetilde{g}_{1}(x)=-\left\langle a_{1}, g(x)\right\rangle=-g_{1}(x)= \begin{cases}x_{1}^{2}\left(\sin \frac{1}{x_{1}}-1\right)-2 x_{1}, & \text { if } \quad x_{1} \neq 0, \\
0, & \text { if } \quad x_{1}=0,\end{cases} \\
& \widetilde{g}_{2}(x)=-\left\langle a_{2}, g(x)\right\rangle=g_{2}(x)=x_{2}^{2}-\left|x_{2}\right|+\frac{3}{2} x_{2} .
\end{aligned}
$$

We have

$$
\begin{aligned}
M & =\left\{x \in C: \widetilde{g}_{i}(x) \leqslant 0(i=1,2), h(x)=0\right\} \\
& =\left\{\left(x_{1}, x_{2}\right) \in[0,1] \times[-1,0]: x_{2}=-\frac{2}{3} x_{1}\right\} .
\end{aligned}
$$

The point $\bar{x}=(0,0)$ is a weakly efficient solution of the vector variational inequality problem:

$$
T(\bar{x})(x-\bar{x}) \notin-\operatorname{int} Q(\forall x \in M) .
$$

It can be seen that

$$
\begin{aligned}
& \partial^{*} \widetilde{g}_{1}(\bar{x})=\{-3,-1\} \times\{0\}, \\
& \partial^{*} \widetilde{g}_{2}(\bar{x})=\{0\} \times\{1 / 2,5 / 2\}, \\
& \partial^{*} h(\bar{x})=\{(1 / 3,1 / 2)\}, \\
& Q^{*}=\left\{\left(x_{1}, x_{2}\right) \in \mathbb{R}^{2}:\left|x_{2}\right| \leqslant-\frac{3}{4} x_{1}\right\}, \\
& T_{C}(\bar{x})=\left\{\left(x_{1}, x_{2}\right) \in \mathbb{R}^{2}: x_{1} \geqslant 0, x_{2} \leqslant 0\right\}, \\
& N_{C}(\bar{x})=\left\{\left(x_{1}, x_{2}\right) \in \mathbb{R}^{2}: x_{1} \leqslant 0, x_{2} \geqslant 0\right\} .
\end{aligned}
$$


It can be seen that (MFCQ) is fulfilled at $\bar{x}=(0,0)$ with $v_{0}=(3,-2) \in T_{C}(\bar{x})$. Moreover, $\bar{x}=(0,0)$ is a regular point in the sense of Ioffe for $h$ relative to $C$, where

$$
H=\left\{\left(x_{1}, x_{2}\right) \in \mathbb{R}^{2}: x_{2}=-\frac{2}{3} x_{1}, 0 \leqslant x_{1} \leqslant 1\right\} .
$$

Thus, all hypotheses of Theorem 4.1 are satisfied, and the necessary condition (3.6) holds for $\bar{\lambda}_{1}=$ $-5, \bar{\lambda}_{2}=1, \bar{\mu}_{1}=1 / 3, \bar{\mu}_{2}=-1 / 3, \bar{v}=-1$.

\section{SUFFICIENT CONDITIONS FOR WEAKLY EFFICIENT SOLUTIONS}

Let $f$ be a function defined on $X$ and let $f$ admit an upper convexificator $\partial^{*} f(\bar{x})$. Following [18], function $f$ is said to be asymptotic pseudoconvex at $\bar{x}$ on $C$ if, $\forall x \in C$,

$$
\text { for some } x_{n}^{*} \in \operatorname{conv} \partial^{*} f(\bar{x}), \lim _{n \rightarrow \infty}\left\langle x_{n}^{*}, x-\bar{x}\right\rangle \geqslant 0 \Longrightarrow f(x) \geqslant f(\bar{x}) \text {. }
$$

$f$ is said to be asymptotic quasiconvex at $\bar{x}$ on $C$ if, $\forall x \in C$,

$$
f(x) \leqslant f(\bar{x}) \Longrightarrow \text { for any } x_{n}^{*} \in \operatorname{conv} \partial^{*} f(\bar{x}), \lim _{n \rightarrow \infty}\left\langle x_{n}^{*}, x-\bar{x}\right\rangle \leqslant 0 .
$$

$f$ is said to be asymptotic quasilinear at $\bar{x}$ on $C$ if, $\forall x \in C$,

$$
f(x)=f(\bar{x}) \Longrightarrow \text { for any } x_{n}^{*} \in \operatorname{conv} \partial^{*} f(\bar{x}), \lim _{n \rightarrow \infty}\left\langle x_{n}^{*}, x-\bar{x}\right\rangle=0 .
$$

Next, we give a sufficient condition for weak efficient solutions of Problem (VVI).

Theorem 5.1. Let $\bar{x} \in M$. Assume that

(i) There exist $\bar{\lambda}:=\left(\bar{\lambda}_{1}, \ldots, \bar{\lambda}_{p}\right) \in Q^{*} \backslash\{0\}, \bar{\mu}_{i} \geqslant 0\left(\forall i \in I(\bar{x}), \bar{\gamma}_{j} \in \mathbb{R}, i=1, \ldots, l\right)$ such that

$$
0 \in \operatorname{cl}\left(\sum_{k \in J} \bar{\lambda}_{k} T(\bar{x})_{k}+\sum_{i \in I(\bar{x})} \bar{\mu}_{i} \operatorname{conv} \partial^{*} \widetilde{g}_{i}(\bar{x})+\sum_{j \in L} \bar{\gamma}_{j} \operatorname{conv} \partial^{*} h_{j}(\bar{x})+N_{C}(\bar{x})\right) .
$$

(ii) Each function $g_{i}$ is asymptotic quasiconvex at $\bar{x}$ on $M(\forall i \in I(\bar{x}))$; each function $h_{j}$ is asymptotic quasilinear at $\bar{x}$ on $M(\forall j \in L)$ and $C$ is convex.

Then $\bar{x}$ is a weak efficient solution of (VVI).

Proof. From (5.1), we see that there exist $\xi_{i}^{(n)} \in \operatorname{conv} \partial^{*} \widetilde{g}_{i}(\bar{x}), \forall i \in I(\bar{x}), \eta_{j}^{(n)} \in \operatorname{conv} \partial^{*} h_{j}(\bar{x}), \forall j \in L$ and $\zeta^{(n)} \in N_{C}(\bar{x})$ such that

$$
0=\lim _{n \rightarrow \infty}\left[\sum_{k \in J} \bar{\lambda}_{k} T(\bar{x})_{k}+\sum_{i \in I(\bar{x})} \bar{\mu}_{i} \xi_{i}^{(n)}+\sum_{j \in L} \bar{\gamma}_{j} \eta_{j}^{(n)}+\zeta^{(n)}\right] .
$$

Hence, for all $x \in M$,

$$
\begin{aligned}
0=\lim _{n \rightarrow \infty} & {\left[\sum_{k \in J} \bar{\lambda}_{k}\left\langle T(\bar{x})_{k}, x-\bar{x}\right\rangle+\sum_{i \in I(\bar{x})} \bar{\mu}_{i}\left\langle\xi_{i}^{(n)}, x-\bar{x}\right\rangle\right.} \\
+ & \left.\sum_{j \in L} \bar{\gamma}_{j}\left\langle\eta_{j}^{(n)}, x-\bar{x}\right\rangle+\left\langle\zeta^{(n)}, x-\bar{x}\right\rangle\right] .
\end{aligned}
$$

Observe that, for all $x \in M$,

$$
g_{i}(x) \leqslant 0=g_{i}(\bar{x}), \quad \forall x \in I(\bar{x}) .
$$

Sine $g_{i}$ is asymptotic quasiconvex at $\bar{x}$, we obtain

$$
0=\lim _{n \rightarrow \infty}\left\langle\xi_{i}^{(n)}, x-\bar{x}\right\rangle \leqslant 0 .
$$


Sine $h_{j}(x)=0=h_{j}(\bar{x}), \forall x \in M$ and $h_{j}, \forall j \in L$ is asymptotic quasilinear at $\bar{x}$, for all $x \in M$, we have

$$
0=\lim _{n \rightarrow \infty}\left\langle\eta_{j}^{(n)}, x-\bar{x}\right\rangle=0 .
$$

Sine $C$ is convex, we have $x-\bar{x} \in T_{C}(\bar{x}), \forall x \in C$. It follows that

$$
0=\lim _{n \rightarrow \infty}\left\langle\zeta^{(n)}, x-\bar{x}\right\rangle \leqslant 0 .
$$

Sine $T(\bar{x})($.$) is continuous linear, it is strictly differentiable and locally Lipschitz on M$. Hence, functions $T(\bar{x})_{k}($.$) have upper convexificators at \bar{x}$ is $\left\{T(\bar{x})_{k}\right\}(\forall k \in J)$. Combining (5.3)-(5.6), for all $x \in M$, we get

$$
0=\lim _{n \rightarrow \infty} \sum_{k \in J} \bar{\lambda}_{k}\left\langle T(\bar{x})_{k}, x-\bar{x}\right\rangle \geqslant 0 .
$$

By virtue of the asymptotic pseudoconvexity of $\bar{\lambda} T(\bar{x})():.=\sum_{k \in J} \bar{\lambda}_{k} T(\bar{x})_{k}($.$) at \bar{x}$ on $M$, it follows from (5.7) that

$$
\bar{\lambda} T(\bar{x}) x \geqslant \bar{\lambda} T(\bar{x}) \bar{x}
$$

which implies that $\bar{x}$ is weakly efficient solutions of (VVI). This completes the proof.

\section{CONCLuSions}

In the paper, we studied nonsmooth vector variational inequality problems with a cone-constraint in which the cone is a polyhedral convex set. Note that weakly efficient solutions are considered with respect to pointed, closed and convex cones. Making use of some results by Luu 4.3 and JeyakumarLuc 4.1, Fritz John and Karush-Kuhn-Tucker necessary conditions for weakly efficient solutions of nonsmooth vector variational inequality problems in terms of convexificators are derived. Under suitable assumptions on the generalized convexity, necessary efficiency conditions become sufficient efficiency ones. The results obtained in this paper can be viewed as extensions of those in [10].

\section{Acknowledgments}

This research was supported by Vietnam National Foundation for Science and Technology Development (NAFOSTED) under Grant No. 101.01-2017.301.

\section{REFERENCES}

[1] Q.H. Ansari, E. Kobis, J.C. Yao, Vector Variational Inequalities and Vector Optimization: Theory and Applications, Springer International, 2017.

[2] J.W. Chen, E. Kobis, M.A. Kobis, J.C. Yao, Optimality conditions for solutions of constrained inverse vector variational inequalities by means of nonlinear scalarization, J. Nonlinear Var. Anal. 1 (2017), 145-158.

[3] F.H. Clarke, Optimization and Nonsmooth Analysis, Wiley, New York, 1983.

[4] V.F. Demyanov, Convexification and concavification of positively homogeneous functions by the same family of linear functions. Technical Report, Univ. of Pisa, No. 3.208.802, pp. 1-11, 1994.

[5] F. Giannessi, G. Mastroeni, L. Pellegrini, On the theory of vector optimization and variational inequalities, image analysis and separation, In: F. Giannessi (ed.) Vector Variational Inequalities and Vector Equilibria: Mathematical Theories, Kluwer, Dordrecht, pp. 153-215, 2000.

[6] X.H. Gong, Scalarization and optimality conditions of vector equilibrium problems, Nonlinear Anal. 73 (2010), 35983612.

[7] M. Golestani, S. Nobakhtian, Convexficators and strong Kuhn-Tucker conditions, Comput. Math. Appl. 64 (2012), 550557. 
[8] A.D. Ioffe, Necessary and sufficient conditions for a loca minimum. 1: A reduction theorem and first order conditions, SIAM J. Control Optim. 17 (1979), 245-250.

[9] V. Jeyakumar, D.T. Luc, Nonsmooth calculus, minimality and monotonicity of convexificators, J. Optim. Theory Appl. 101 (1999), 599-612.

[10] D.V. Luu, D.D. Hang, On optimality conditions for vector variational inequalities, J. Math. Anal. Appl. 412 (2014), 792-804.

[11] D.V. Luu, Optimality condition for local efficient solutions of vector equilibrium problems via concavificators and applications, J. Optim. Theory Appl. 171 (2016), 643-665.

[12] D.V. Luu, Convexficators and necessary conditions for efficiency, Optimization 63 (2014) 321-335.

[13] D.V. Luu, Necessary and sufficient conditions for efficiency via convexificators, J. Optim. Theory Appl. 160 (2014), 510-526.

[14] J. Morgan, M. Romaniello, Scalarization and Kuhn-Tucker like conditions for weak vector generalized quasivarationl inequalities, J. Optim. Theory Appl. 130 (2006), 309-316.

[15] P. Michel, J.P. Penot, Calcul sous-differentiel pour des fonctions lipschitziennes et nonlipschitziennes, C. R. Math. Acad. Sci. 12 (1984), 269-272

[16] B.S. Mordukhovich, Y. Shao, On nonconvex subdifferential calculus in Banach spaces, J. Convex Anal. 2 (1995), $211-228$.

[17] W. Schirotzek, Nonsmooth Analysis, Springer, Berlin Heidelberg New York, 2007.

[18] X.Q. Yang, Continuous generalized convex functions and their characterizations, Optimization 54 (2005), $495-506$.

[19] D.E. Ward, G.M. Lee, On relations between vector optimization problems and vector variational inequalities, J. Optim. Theory Appl. 113 (2002), 583-596. 